\title{
Successful treatment of angiosarcoma of the scalp with apatinib: a case report
}

\author{
This article was published in the following Dove Press journal: \\ OncoTargets and Therapy \\ II August 2016 \\ Number of times this article has been viewed
}

\author{
Guanghui Ji' \\ Liu Hong ${ }^{2}$ \\ Ping Yang ${ }^{3}$ \\ 'Department of Traditional Chinese \\ Medicine, ${ }^{2}$ Department of Pathology, \\ ${ }^{3}$ Department of Oncology, PLA Navy \\ General Hospital, Beijing, People's \\ Republic of China
}

\begin{abstract}
Angiosarcoma is a rare and aggressive vascular malignancy with a poor prognosis. There is no standard chemotherapy regime for advanced angiosarcoma. Here, we report a case of advanced angiosarcoma that was successfully treated with apatinib, an oral tyrosine kinase inhibitor targeting the intracellular domain of vascular endothelial growth factor receptor-2 (VEGFR-2). To our knowledge, this is the first case to report the successful use of apatinib for angiosarcoma. Furthermore, the administration of apatinib results in fewer toxic effects than traditional cytotoxic chemotherapy. Quantitative polymerase chain reaction analysis revealed high expression of VEGFR-2 mRNA, suggesting that apatinib leads to clinical response by inhibiting VEGFR-2 tyrosine kinase activity and that VEGFR-2 plays a crucial role for angiosarcoma. Apatinib may be an additional option for angiosarcoma especially for the aged and patients with poor performance status. Further prospective studies are required to optimize the use of apatinib in patients with angiosarcoma and to identify which patients will benefit from the agent.
\end{abstract}

Keywords: hemangiosarcoma, VEGFR-2, chemotherapy, angiogenesis inhibitor, targeted therapy

\section{Introduction}

Angiosarcomas are rare aggressive sarcomas derived from vascular endothelial cells. Currently, standard treatment guidelines for angiosarcoma do not exist. ${ }^{1}$ The prognosis is poor with a reported 5-year survival rate of 35\% in nonmetastatic cases and a recurrence rate of up to $75 \%$ within 2 years of local treatment. ${ }^{2}$ Surgery could be the most effective method of treatment, but for advanced and metastatic angiosarcoma, chemotherapy would be the main choice. Considering that the prevalence of angiosarcomas is high in elderly patients aged between 70 and 75 years $^{3}$ and that the toxicities associated with combined chemotherapy often require treatment cessation, patients with angiosarcomas require newer, safer, and more effective treatments.

We report a case of angiosarcoma of the scalp, wherein the patient exhibited partial response (PR) to apatinib 4 (Hengrui Pharmaceutical Co., Ltd, Shanghai, People's Republic of China). To the best of our knowledge, this is the first case of angiosarcoma of the scalp that responded to apatinib. This case report was approved by the Medical Ethics Committee of the Navy General Hospital, and written informed consent was obtained from the patient for publication of this case report and accompanying images.

\section{Case report}

In November 2014, a 74-year-old male came to our hospital because of an irregular soft tissue mass on the scalp with an area of $4.8 \times 1.3 \mathrm{~cm}$.

The presence of distal metastasis was ruled out by enhanced brain computed tomography (CT) and chest/abdomen/pelvic CT, and complete resection was 
performed on November 21, 2014. The pathology diagnosis was angiosarcoma (Figure 1A and B) with immunophenotype: AE1/AE3(-), CD31(1+), CD34(-), CK18(-), F8(-), $\operatorname{Vim}(3+)$, CK5\&6(-), P63(-), EMA(-), GCDFP 15(-), and Ki-67 (20\%).

Two months after surgery, multiple nodules appeared around the operation area, indicating local recurrence. The patient was then referred to a radiation oncologist to receive radiotherapy with a total of 60 Gy of intensity-modulated radiation therapy.

During radiotherapy, a nodule of diameter $2 \mathrm{~cm}$ was found in the right temporal scalp near the edge of the previous surgery area. Whole-body positron emission tomography/CT confirmed local tumor recurrence with multiple metastases in the right temporal lobe, subcutaneous adipose tissue, and bilateral lung lobar.

Cytotoxic chemotherapy was not given because of his old age and poor performance status (PS). Quantitative polymerase chain reaction of vascular endothelial growth factor receptor-2 (VEGFR-2) mRNA was investigated and the result revealed high expression of VEGFR-2 mRNA.

After the patient provided written informed consent, apatinib was administered at a dose of $500 \mathrm{mg} /$ day. Following 1 month of therapy, multiple lung metastases (Figure 2A-C) were either significantly reduced in size (Figure 2D, as indicated by arrowheads) or disappeared (Figure 2E and F), and the recurrent tumors of the scalp also disappeared, which was considered to be a PR. The patient then continued to use apatinib as maintenance therapy. The disease progressed in March 2016, with new lung metastases by enhanced chest CT and multiple scalp nodules by enhanced brain magnetic resonance imaging (MRI), which was considered to be a relapse. The progression-free survival time was 12 months. The only toxicity was mild hand-foot syndrome.

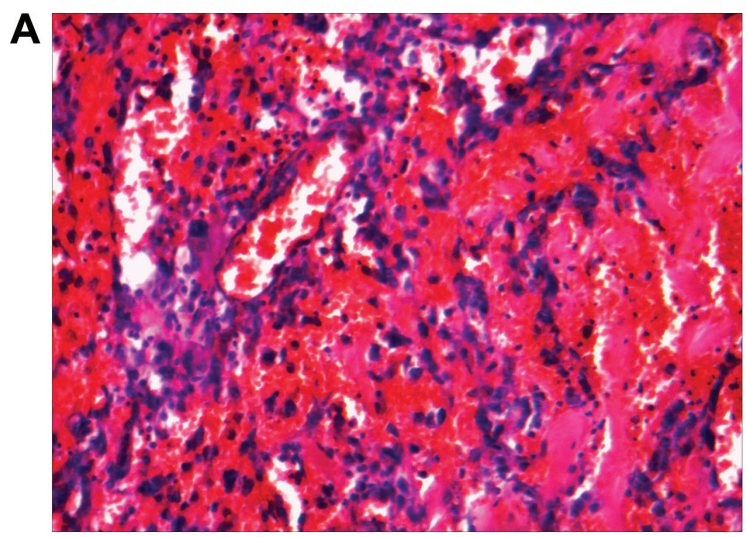

Figure I Hematoxylin and eosin (H\&E) staining of a tumor section. Notes: (A) $\times 200$; (B) $\times 400$.

\section{Discussion}

Angiosarcomas are malignant mesenchymal neoplasms of vascular endothelial cells. The face and scalp regions account for more than $50 \%$ of cutaneous angiosarcomas. ${ }^{2}$

Treatment modalities for angiosarcoma include resection with a wide margin, radiotherapy, and chemotherapy. However, even for a local disease, clear margins account for only $\sim 20 \%-35.6 \%$ of scalp angiosarcoma. For this reason, combination treatment is recommended to obtain a better prognosis than any single regimen. ${ }^{5}$

For metastatic angiosarcomas, cytotoxic chemotherapy would be the main treatment. However, newer, safer, and more effective treatments are needed because of the toxicities associated with chemotherapy, especially for the aged.

It has been reported that angiosarcomas overexpress not only VEGF proteins but also VEGFRs, which implies that both VEGF and VEGFR might be the treatment targets for this type of tumor. ${ }^{6,7}$ VEGFR-2 has been reported to be the primary VEGF family receptor driving angiogenesis. It could bind all VEGF-A isoforms, VEGF-C, and VEGF-D. The binding of VEGF-A to VEGFR-2 induces cellular proliferation and endothelial cell survival through a cascade of different signaling pathways such as PLC- $\gamma$-Raf kinaseMEK-MAP kinase and PI3K-AKT pathways. ${ }^{8}$

Apatinib is a novel small molecule tyrosine kinase inhibitor targeting the intracellular domain of VEGFR-2. With a favorable side-effect profile and improved outcomes, apatinib has been shown to be a new therapeutic option in a variety of tumor types. ${ }^{4,9}$ However, successful use of apatinib in angiosarcomas has not been reported.

In Phase I study of apatinib, dose escalation was conducted from 250 to $1,000 \mathrm{mg}$ in patients aged between 18 and 70 years. Maximum tolerated dose was determined to be $850 \mathrm{mg}$ once daily. The recommended dose was $750 \mathrm{mg}$ once

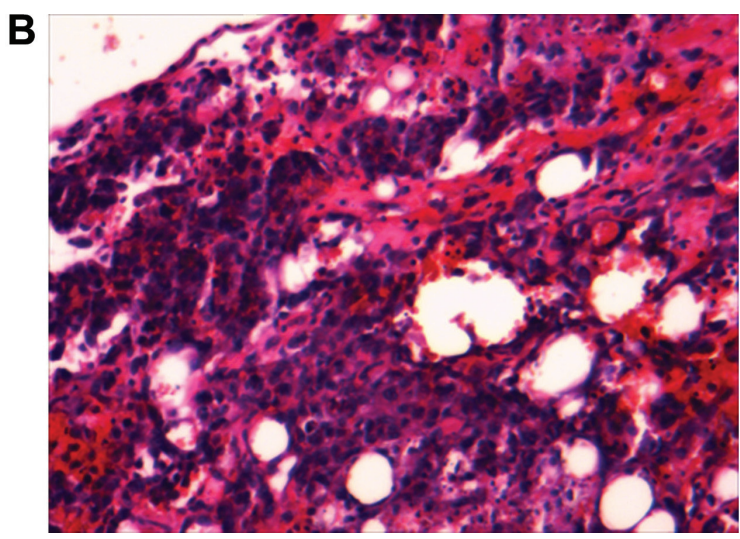



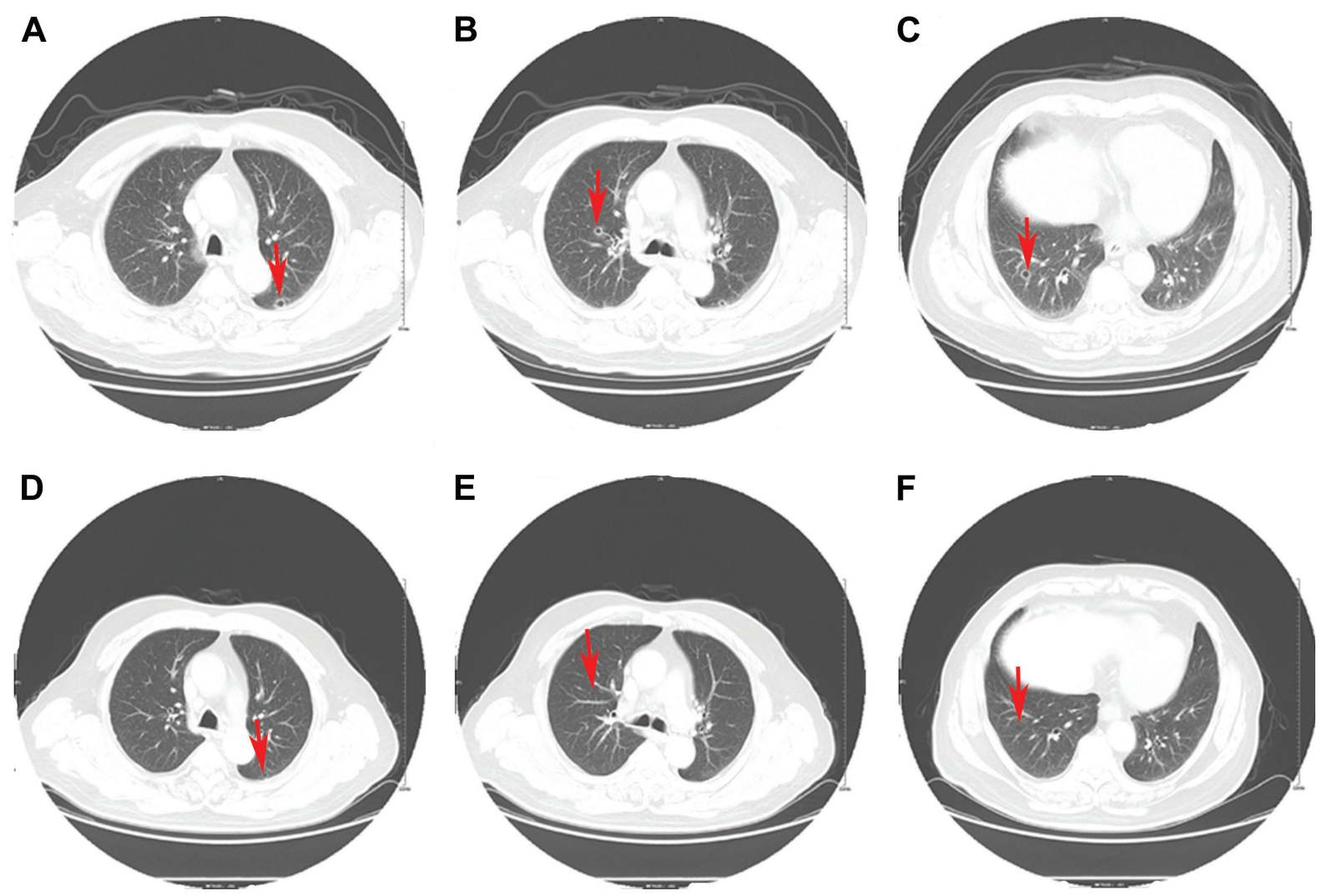

Figure $\mathbf{2}$ Chest CT images showing metastases before $(\mathbf{A}-\mathbf{C})$ and after (D-F) treatment with apatinib.

Note: The arrowheads indicate the pulmonary metastases.

Abbreviation: CT, computed tomography.

daily. The most frequently observed drug-related adverse events were hypertension (69.5\%), proteinuria (47.8\%), and hand-foot syndrome $(45.6 \%) .^{10}$

In this case report, the patient with angiosarcoma developed local recurrence and multiple lung metastases shortly after surgery. Owing to old age and poor PS, the patient could not tolerate toxic chemotherapy, and apatinib monotherapy was then used to control the disease. Considering his old age, being more than 70 years, and medical history of hypertension, a dose of $500 \mathrm{mg}$ was given. The disease was controlled successfully with a PR and a progression-free survival time of 12 months. In addition, the administration of apatinib resulted in no major toxic profiles except for mild hand-foot syndrome.

Gene expression analysis revealed high expression of VEGFR-2 mRNA, suggesting that apatinib led to clinical response by inhibiting VEGFR-2 tyrosine kinase activity.

\section{Conclusion}

Apatinib may provide an additional option for the treatment of angiosarcomas, especially for the aged and patients with poor PS. Further prospective studies are required to optimize the use of apatinib in the treatment of angiosarcoma and to identify which patients will benefit from the agent.

\section{Acknowledgment}

The authors thank the patient for his participation and his agreement to the publication of the report.

\section{Author contributions}

All authors contributed toward data analysis, drafting and critically revising the paper, gave final approval of the version to be published, and agreed to be accountable for all aspects of the work.

\section{Disclosure}

The authors report no conflicts of interest in this work.

\section{References}

1. Young RJ, Brown NJ, Reed MW, Hughes D, Woll PJ. Angiosarcoma. Lancet Oncol. 2010;11(10):983-991.

2. Ye J, Li XF, Wang YD, Yuan Y. Long-term survival of a patient with scalp angiosarcoma and multiple metastases treated using combination therapy: a case report. Oncol Lett. 2015;9(4):1725-1728.

3. Ono S, Tanioka M, Fujisawa A, Tanizaki H, Miyachi Y, Matsumura Y. Angiosarcoma of the scalp successfully treated with a single therapy of sorafenib. Arch Dermatol. 2012;148(6):683-685. 
4. Scott AJ, Messersmith WA, Jimeno A. Apatinib: a promising oral antiangiogenic agent in the treatment of multiple solid tumors. Drugs Today (Barc). 2015;51(4):223-229.

5. Hwang K, Kim MY, Lee SH. Recommendations for therapeutic decisions of angiosarcoma of the scalp and face. J Craniofac Surg. 2015; 26(3):e253-e256.

6. Stacher E, Gruber-Mösenbacher U, Halbwedl I, et al. The VEGF-system in primary pulmonary angiosarcomas and haemangioendotheliomas: new potential therapeutic targets? Lung Cancer. 2009;65(1):49-55.

7. Tokuyama W, Mikami T, Masuzawa M, Okayasu I. Autocrine and paracrine roles of VEGF/VEGFR-2 and VEGF-C/VEGFR-3 signaling in angiosarcomas of the scalp and face. Hum Pathol. 2010;41(3):407-414.
8. Tian S, Quan H, Xie C, et al. YN968D1 is a novel and selective inhibitor of vascular endothelial growth factor receptor-2 tyrosine kinase with potent activity in vitro and in vivo. Cancer Sci. 2011;102(7): 1374-1380.

9. Ji G, Hong L, Yang P. Successful treatment of advanced malignant fibrous histiocytoma of the right forearm with apatinib: a case report. Onco Targets Ther. 2016;9:643-647.

10. Li J, Zhao X, Chen L, et al. Safety and pharmacokinetics of novel selective vascular endothelial growth factor receptor-2 inhibitor YN968D1 in patients with advanced malignancies. BMC Cancer. 2010;10:529.

\section{Publish your work in this journal}

OncoTargets and Therapy is an international, peer-reviewed, open access journal focusing on the pathological basis of all cancers, potential targets for therapy and treatment protocols employed to improve the management of cancer patients. The journal also focuses on the impact of management programs and new therapeutic agents and protocols on

\section{Dovepress}

patient perspectives such as quality of life, adherence and satisfaction The manuscript management system is completely online and includes a very quick and fair peer-review system, which is all easy to use. Visit http://www.dovepress.com/testimonials.php to read real quotes from published authors. 